\title{
Data Quality Artificial Intelligence for Data Classification
}

\author{
Naveen Kumar S R, Dr. G Mahadevan and S.Vidivelli
}

\begin{abstract}
Outlier detection is a critical issue of records excellent manipulate because it permits analysts and engineers the ability to become aware of facts first-rate troubles through using their very own data as a device. However, conventional information high-quality manipulate strategies are based totally on users' experience or previously set up business policies, and this boundaries performance similarly to being a completely time ingesting method and low accuracy. Utilizing massive records, we can leverage computing sources and advanced strategies to overcome these challenges and provide greater fee to the business.

In this paper, we first overview applicable works and discuss gadget mastering strategies, gear, and statistical nice models. Second, we provide a creative records profiling framework primarily based on deep Studying and statistical model algorithms for improving statistics first-class. Third, authors use public Arkansas officers' salaries, one of the open datasets to be had from the state of Arkansas' authentic internet site, to illustrate the way to become aware of outlier statistics for improving records pleasant thru machine studying. Finally, we talk future works.
\end{abstract}

Keywords - statistics first-rate, information smooth, deep gaining knowledge of, statistical best manipulate, information profiling.

\section{INTRODUCTION}

Digital transformation is actual, and it's miles upon us. It's a rely of "disrupt or be disrupted." Organizations are using transformative projects to enhance monetary universal overall performance and aggressive characteristic in their industries.

Examples of those projects embody deepening customer relationships, optimizing operations, personalizing healthcare, and preventing fraud. The key aspect using the success of those tasks is the capability to gas them with trusted and nicely timed statistics. It's quite smooth: Successful digital strategies are constructed on information. The competence you assemble round statistics control will determine how a success your virtual technique is. Or, located some other manner, your virtual technique may be most effective as effective as the data that informs it. Odds are, but that dealing with statistics "the way you constantly have" receivers lessen it. IT leaders are seeking out ways to enhance information control productiveness to make higher records available to all, faster. Artificial intelligence (AI) and system-studying strategies powered via company-huge information and metadata, will

Naveen Kumar S R, Research Scholar, Department of Computer Science, Rayalaseema University, Kurnool, India.

Dr. G Mahadevan, Principal, Annai College of Engineering, Kumbakonam, India.

S.Vidivelli, Assistant Professor, Annai College of Engineering, Kumbakonam, India. significantly boost the productivity of all managers and users of information throughout the business enterprise.

\section{RELATED WORK}

Outlier statistics manner this information is totally distinct from the others. Hawkins formally defined it as "An outlier is a commentary which deviates so much from the alternative observations as to arouse suspicions that it was generated by means of a one-of-a-kind mechanism". However, outlier facts do no longer suggest errors records or hassle data, however this facts way it has ability error records or chance information. Outlier detections could be utilized in distinct industries. For example, mentions that intrusion detection structures, credit card fraud, thrilling sensor occasions, scientific prognosis, law enforcement, and earth technology can make use of this technology.

According to, there are many outlier detections supporting human beings to perceive outlier information, which includes probabilistic and statistical models, linear correlation analysis, proximity-primarily based detection, and supervised outlier detection.

\section{WHAT IS MACHINE LEARNING?}

Machine learning is a technique wherein applications iteratively analyze from information in place of being static. Machine learning structures are used to build an input-based totally model that can be used to make predictions or decisions. These structures study from the records and may modify themselves consequently to provide better results. The greater statistics they've, the faster they analyze and the extra correct their effects.

\section{WHY SYSTEM GETTING TO KNOW FOR RECORDS CONTROL?}

To scale up the rate of information shipping for essential commercial enterprise tasks, you need to increase automation. That is wherein system studying comes in. With enterprise-wide metadata visibility and device mastering, records control gear can be "taught" to make sensible guidelines and to automate many statistics control tasks. Machine gaining knowledge of does no longer update records analysts and different customers; as a substitute, it is key to growing the productivity and effectiveness of the fact's management team of workers within an organization.

Machine getting to know may be used to improve duties which are tedious or impossible to do at human scale. Some examples encompass: 
1. Discovery and identity

- Data best regulations, and commercial enterprise entity discovery

- Semantic seek, pattern identification, and facts classification,

- Anomaly detection and notification

2. Predictive operations

- Burst to deal with information spikes

- Prioritize operational problem investigations

- Self-heal to handle changes to environments

\section{Next-first-rate-movement \& hints}

- Suggest statistics units, transforms, and rules

- Auto-map, cleanse, and standardize from sources to goal

- Self-combine new sources of information

The foundation for system mastering in information control

Effective machine mastering requires large schooling statistics units. In a statistics management context, the satisfactory supply

of statistics is an organization-extensive records catalog. Most companies have thousands of databases, data files, programs, and analytics systems. By harvesting the metadata across these information repositories, organizations can build a richly populated catalog. The combination of gadget gaining knowledge of and a data catalog with enterprise wide visibility into metadata would provide the idea for intelligence that might make a in reality significant and high-quality impact on facts control productiveness. In this era of cloud, it's far essential to word that this method works for SaaS applications as well. Metadata can be amassed from SaaS packages along with Sales force and Workday and delivered to the enterprise catalog.

\section{OVERVIEW ARCHITECTURE}

The Intelligent Data Platform (IDP): We have delivered an integrated stop-to-give up records management platform for max productivity. By offering unified connectivity, metadata, and operations control, the unified platform quickens the improvement and deployment of new records management projects. The platform presents a effective and regular set of talents for handling facts throughout on-premises, cloud, and large facts resources. We name this unified fact management platform the Intelligent Data Platform.

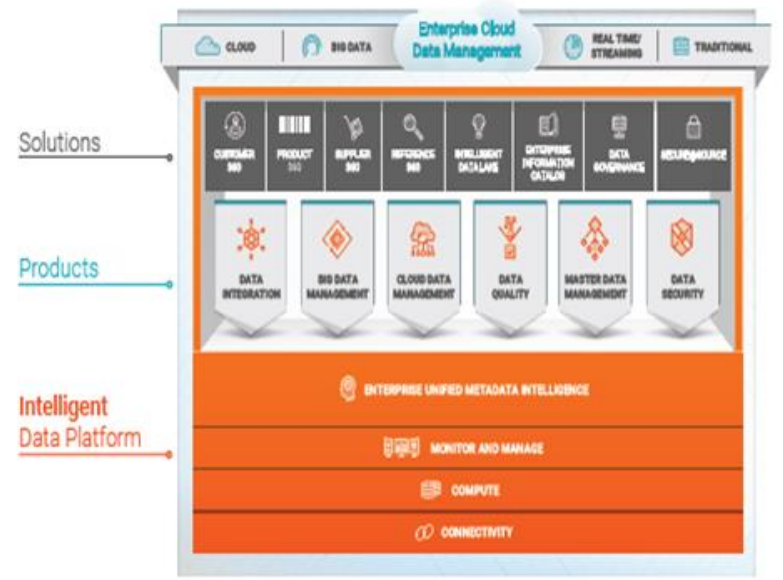

Metadata: Has long been referred to as a pacesetter for its management of technical and commercial enterprise metadata. Elevated its abilities in this region by way of collecting a broader spectrum of metadata from across the enterprise, along with:

- Technical metadata, inclusive of database tables, column data, and records profile information

- Business metadata, which captures context approximately records, its meaning, relevance, and criticality to numerous business techniques and capabilities Operational metadata, approximately systems and process execution, inclusive of while turned into the data remaining up to date?

When changed into the load system final run? Which records changed into most accessed?

- Usage metadata, about user activity, which include information sets accessed, seek effects clicked on, ratings or feedback furnished this broader series of metadata is important to machine gaining knowledge of. It presents statistics sets which are used to "train" the system mastering algorithms and allows them to permits them to regulate to produce higher outcomes.

\section{INTELLIGENT ENTITY DISCOVERY}

Once domain names for columns had been diagnosed, system can collect those character fields into better-level business entities. The example under shows how an entity referred to as Purchase Order is created by combining fields identified as Customer and as Product. Entity discovery learns from how customers have assembled disparate statistics fields in their analytics or statistics integration strategies and applies this mastering to derive entities throughout the enterprise facts panorama. 


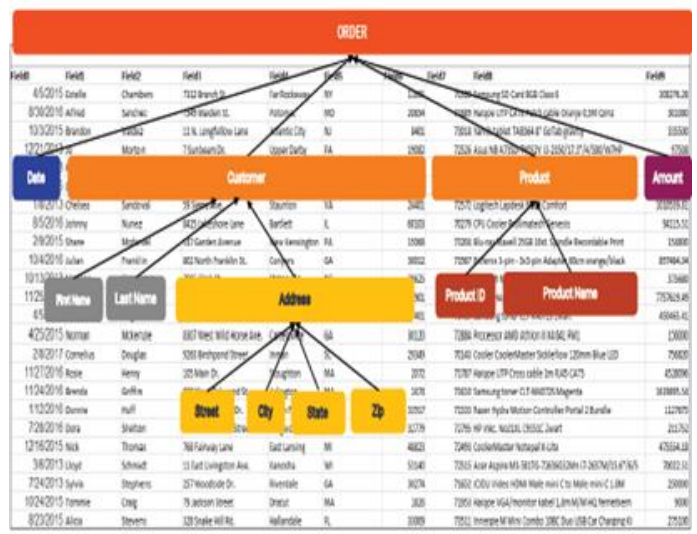

\section{INTELLIGENT STRUCTURE DISCOVERY}

Intelligent structure discovery uses a genetic algorithm to automate the popularity of patterns within the files. In this technique, it makes use of the concept of "evolution" to improve consequences. Each candidate solution has a fixed of houses that can be altered and then tested to decide if they provide an answer with a higher healthy. It neither requires person enter to define the structure of the record nor is unique to a fixed of industry record formats. Initial structures of the file are derived primarily based on fundamental delimiter-primarily based parsing. These structures are then scored on the basis of several elements, which include enter insurance and derived domains. Top scored systems input a "mutation" segment where numerous adjustments are made to the systems, as an example, combining substructures to see if the rankings improve. It terminates the process while it determines suitable health of the structure to the records.

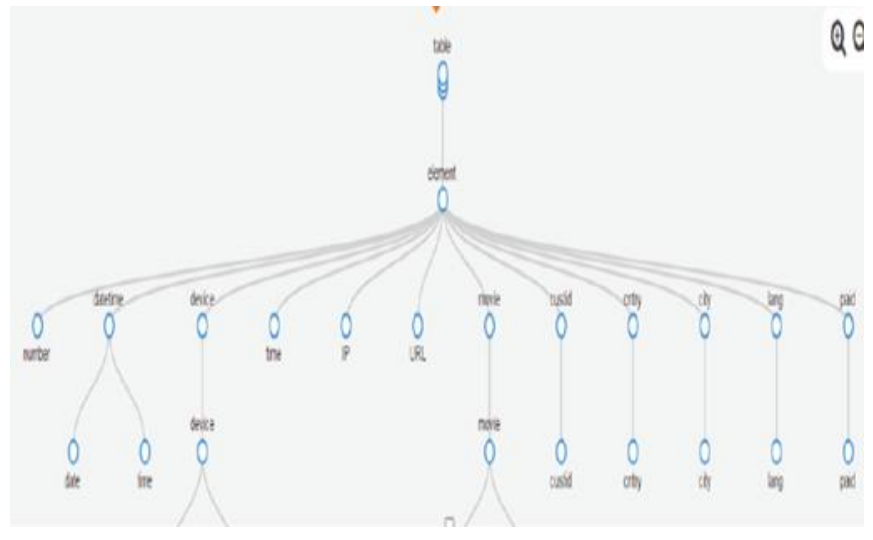

\section{CONCLUSION}

Today's information-centric commercial enterprise techniques are built on a foundation of information. Winning requires constructing a competence in data control to unharness the power of information. With all demanding situations that statistics management gives underneath regular circumstances,

Traditional techniques can't scale to satisfy these day's necessities - to say nothing of the following days. One way to leverage your records to drive disruption is to standardize on information management platform that makes use of the strength of information, metadata, and machine mastering/AI to decorate the productivity of all customers of the platform: technical, operational, business, and specifically business self-carrier.

\section{FUTURE WORK}

Data profiling of records fine could be very vital for big data. Deep mastering is a very promising method for doubtlessly solving many huge facts challenges. We will keep discovering different neural community fashions with numerous datasets. We will even conduct those experiments on GPU-based totally server to expedite the deep getting to know performance, and are trying to find powerful data visualization solutions.

\section{REFERENCES}

[1] Redman, T. (2001), Data Quality: The Field Guide, Publisher: Digital Press, Newton, Massachusetts, USA.

[2] Savchenko, S. (2003), Automating objective data quality assessment, 8th InternationalConference on Information Quality, Boston, Massachusetts, USA.

[3] Shankaranarayan, G., Ziad, M. and Wang, R.Y. (2003). Managing data quality in dynamic decision environments: an information product approach, Journal of DataManagement, 14(4)pp.14-32. https://doi.org/10.4018/jdm.2003100102

[4] Slone (2006), Information Quality Strategy: An Empirical Investigation of the Relationshipbetween Information Quality Improvements and Organizational Outcomes,Ph.D. Dissertation. Capella University.

[5] Stvilia, B., Gasser, L., Twidale, M.B. and Smith, L.C. (2007), A framework for information quality assessment, Journal of the American Society for Information Scienceand Technology. 58(12), pp. 1720-1733. https://doi.org/10.1002/asi.20652

[6] 7. Kahn, B.K., Strong, D.M., and Wang, R.Y. Information Quality Benchmarks: Product andService Performance. Commun. ACM, (2002). https://doi.org/10.1145/505999.506007

[7] Cappiello, C., Francalanci, C., \&Pernici, B. (2004) Data quality assessment from user 's perspective. Procedures of the 2004 International Workshop on Information Quality in Information Systems, New York: ACM, pp 78-73. https://doi.org/10.1145/1012453.1012465

[8] Science (2011) Special online collection: Dealing with data. Retrieved November 5, 2013 from the World Wide Web: http://www.sciencemag.org/site/special/data/

[9] Wang, J. L., Li, H., \& Wang, Q. (2010) Research on ISO 8000 Series Standards for Data Quality. Standard Sciาence 12, pp 44-46.

[10] Wang, R., \&Storey, V. (1995) Framework for Analysis of Quality Research. IEEE Transactions on Knowledge and Data Engineering 1(4), pp 623-637.

https://doi.org/10.1109/69.404034 